\title{
Effectiveness of diabetes in pregnancy study group India diagnostic criterion in detecting gestational diabetes mellitus: a rural Bangalore study
}

\author{
Rajiv Kumar Saxena, Noor Fathima Tameem Ansari*, Pallavi Singh
}

Department of Obstetrics and Gynecology, MVJ Medical College and Research Centre, Bangalore, Karnataka, India

Received: 18 November 2019

Revised: 07 December 2019

Accepted: 30 December 2019

\section{*Correspondence:}

Dr. Noor Fathima Tameem Ansari,

E-mail: noortheglory@gmail.com

Copyright: (C) the author(s), publisher and licensee Medip Academy. This is an open-access article distributed under the terms of the Creative Commons Attribution Non-Commercial License, which permits unrestricted non-commercial use, distribution, and reproduction in any medium, provided the original work is properly cited.

\section{ABSTRACT}

Background: The oral glucose tolerance test (OGTT) is considered the current standard for diagnosis of gestational diabetes mellitus (GDM). International association of diabetes and pregnancy study groups (IADPSG) recommends OGTT, with fasting, one-hour and two-hour venous blood samples drawn after intake of $75 \mathrm{~g}$ oral glucose. In the Indian context, diabetes in pregnancy study group in India (DIPSI) recommends glucose challenge test (GCT), where $75 \mathrm{~g}$ glucose is given irrespective of the fasting state, and a single venous sample is drawn after two-hour. Diagnosis of GDM is made, if any cut-off value is met or exceeded. This prospective study was conducted to compare the DIPSI and IADPSG criteria for diagnosis of GDM.

Methods: Pregnant women between 24 to 28 weeks of gestation were subjected to non-fasting GCT. Capillary sample were also drawn simultaneously using Accu check active glucometer. These women were counselled to undergo a standard 75gram OGTT, within a week of recruitment.

Results: According to IADPSG criteria, 17.6\% (18/102) of our participants had GDM, as compared to $19.6 \%$ (20/102) by DIPSI criteria using venous samples, and $25.3 \%$ (25/99) by capillary sample method. Sensitivity and specificity of GCT using venous sample was $72.22 \%$ and $91.67 \%$, and using capillary sample was $70.59 \%$ and $84.15 \%$ respectively. DIPSI criteria using venous samples wrongly labelled $8.3 \%$ women as GDM, and capillary samples wrongly labelled $15.9 \%$ women as GDM. More importantly DIPSI criteria using venous samples labelled $27.8 \%$ women as false-negative and capillary samples labelled $29.4 \%$ women as false-negative for GDM. Almost one quarter of women with GDM will be missed if DIPSI criteria is used as a universal screening modality.

Conclusions: We suggest that the IADPSG criteria be used for diagnosis of GDM in antenatal women in India.

Keywords: Diabetes in pregnancy study group in India, Gestational diabetes mellitus, International association of diabetes and pregnancy study groups, Oral glucose tolerance test, Pregnancy

\section{INTRODUCTION}

Pregnancy is associated with insulin resistance (IR) and hyperinsulinemia that may predispose some women to develop diabetes mellitus. Gestational diabetes mellitus has been described as any degree of glucose intolerance with an onset, or first recognition during pregnancy. The
International association of diabetes and pregnancy study groups (IADPSG) classify hyperglycemia first detected during pregnancy as either "overt diabetes" or "gestational diabetes mellitus (GDM)". ${ }^{1}$

In 2013, the World Health Organization (WHO) also recommended that hyperglycemia first detected during 
pregnancy be classified as either "diabetes mellitus (DM) in pregnancy" or "GDM". 2

A number of risk factors are associated with the development of GDM. The common risk factors are obesity, older maternal age, past history of GDM, strong family history of diabetes mellitus, member of an ethnic group with a high prevalence of type- 2 diabetes mellitus (T2DM), polycystic ovarian syndrome, and persistent glucosuria. ${ }^{3}$ A history of delivering big baby (birth weight $\geq 4000 \mathrm{~g}$ ), history of recurrent abortions, history of unexplained stillbirths, and history of essential hypertension, or pregnancy-related hypertension are other risk factors for GDM. Screening for GDM is advocated to be done at the first visit if the patient has any high-risk factors as mentioned above. ${ }^{4}$ However, if the results of the first test are normal or the patient does not have any high-risk factors, then the screening test is done during second trimester, i.e. at 24-28 weeks of gestation due to increasing insulin resistance. IADPSG have recommended oral glucose tolerance test (OGTT) as a diagnostic method for GDM. The same was adopted by WHO in $2013 .{ }^{5}$ A pregnant woman is counselled to come for the test after overnight ( 8 hours) fasting. The plasma glucose level is measured in a fasting state and then anhydrous $75 \mathrm{~g}$ glucose is given in a glass $(250-300 \mathrm{ml})$ of water, to drink over 5 to 10 minutes. Plasma glucose level is measured again after 2 hours. During pregnancy, if fasting plasma glucose is $>92 \mathrm{mg} / \mathrm{dl}$ and two-hour value is > $153 \mathrm{mg} / \mathrm{dl}$, gestational diabetes mellitus is diagnosed. As per WHO there are no established criteria for the diagnosis of diabetes based on the 1-hour postload value as advocated by IADPSG.

The diabetes in pregnancy study group India (DIPSI) has established the practice guidelines for GDM for the Indian environment. ${ }^{6}$ Due to high prevalence of GDM, screening is essential for all Indian pregnant women. DIPSI recommends a glucose challenge test (GCT), wherein, a pregnant woman has to be given a $75 \mathrm{~g}$ oral glucose load with a glass $(250-300 \mathrm{ml})$ of water, irrespective of the fasting status. After 2 hours, a venous blood sample is collected for estimating plasma glucose level. The Indian criteria for diagnosis of GDM, as defined by DIPSI, is two-hour plasma glucose level of $140 \mathrm{mg} / \mathrm{dl}$ or more. ${ }^{7}$

This "single-step procedure" was developed due to the practical difficulty in performing glucose tolerance test in the fasting state, as seldom pregnant women visiting the antenatal clinic for the first time come in the fasting state. If they are asked to come on another day in the fasting state many of them do not return. This one step procedure is simple, economical and convenient for the patients. ${ }^{8}$

Dilemma concerning optimal strategy for diagnosis of GDM still remains, with different national and international bodies recommending different guidelines for diagnosis of GDM. ${ }^{9}$ In view of the above, this prospective study was conducted to compare the DIPSI and IADPSG criteria for diagnosis of GDM, in our tertiary care center in rural Bengaluru.

\section{METHODS}

This cross-sectional prospective study was conducted at an antenatal clinic in a tertiary care hospital, in rural Bangalore. 106 pregnant women between 24 to 28 weeks of gestation were recruited by simple random sampling method, on the basis of a predefined inclusion and exclusion criteria. An initial assessment of patient was performed including detailed history (any family history of diabetes, history of previous pregnancies, socioeconomic status etc.), general examination including calculation of body mass index (BMI), and an obstetric examination was performed.

All women who consented for the study were subjected to non-fasting $75 \mathrm{~g} \mathrm{GCT}$ on the same day irrespective of her fasting status. A venous sample was drawn after two hour of glucose intake. Simultaneously, a capillary blood sample of patient was tested, using 'Accu check active' glucometer, from 'Roche diagnostics'. These women were counselled to undergo a standard 75gram OGTT, within a week of recruitment. OGTT was performed after an overnight fasting of at least 8 hours. Fasting sample, one hour and two-hour venous samples were drawn, and plasma glucose measurements were carried out with an automated analyzer using the glucose oxidase enzymatic calorimetric assay. GDM was diagnosed according to standard IADPSG criteria and the diagnosis was made when any of the following thresholds were met or exceeded: Fasting: $\geq 92 \mathrm{mg} / \mathrm{dL}$, one hour: $\geq 180 \mathrm{mg} / \mathrm{dL}$, two hours: $\geq 153 \mathrm{mg} / \mathrm{dL}$. SSPS 21.0 was used for statistical analysis and p-value less than 0.05 was taken as statistically significant.

\section{Inclusion criteria}

- All consenting pregnant women in second trimester between 24-28 weeks.

\section{Exclusion criteria}

- Known pre-gestational diabetes

- Chronic renal/ cardiac/ hepatic/ respiratory diseases

- Taking drugs that alter glucose metabolism.

\section{RESULTS}

A prospective study was carried out among 106 pregnant women between 24-28 weeks of gestation. A total 102 antenatal women were included in the final analysis, 4 patients were excluded as 3 patients did not follow up for OGTT and 1 patient was diagnosed with anomalous fetus at 24 weeks. Capillary blood glucose data was not available for 3 women and hence they were excluded and only 99 women's data was analyzed for DIPSI criteria using capillary blood glucose values. 
The mean age of our study participants was $23.74 \pm 4.02$, minimum age was 18 years and maximum age was 34 years (95\% CI 22.95 to 24.52). The mean of pre- pregnancy body mass index (BMI) was 22.82 \pm 3.52 $\mathrm{kg} / \mathrm{m}^{2}$, minimum BMI was $16 \mathrm{Kg} / \mathrm{m}^{2}$ and maximum was $33 \mathrm{Kg} / \mathrm{m}^{2}$ (95\% CI 22.13 to 23.51 ) (Table 1 ).

Table 1: Age and pre-pregnancy BMI in studied cases.

\begin{tabular}{|lllllll|}
\hline Parameter & Mean \pm SD & Median & Min & Max & 95\% CI & Lower \\
\hline Age & $23.74 \pm 4.02$ & 23.00 & 18.00 & 34.00 & 22.95 & 24.52 \\
\hline Pre-pregnancy BMI & $22.82 \pm 3.52$ & 22.00 & 16.00 & 33.00 & 22.13 & 23.51 \\
\hline
\end{tabular}

Table 2: Prevalence of GDM based on different criteria.

\begin{tabular}{|c|c|c|c|c|}
\hline Criteria & Cut-off & Number (positive) & Percentage & $95 \% \mathrm{CI}$ \\
\hline \multirow{4}{*}{ IADPSG $(n=102)$} & Fasting $\geq 92$ (or) & 1 & \multirow{4}{*}{17.6} & \multirow{4}{*}{$11.5-26.2$} \\
\hline & 1 hour $\geq 180$ (or) & 8 & & \\
\hline & 2 hours $\geq 153$ & 9 & & \\
\hline & $\begin{array}{l}\text { Any one of the above- } \\
\text { mentioned criteria }\end{array}$ & 18 & & \\
\hline DIPSI by venous glucose $(n=102)$ & 2 hours $\geq 140$ & 20 & 19.6 & $13.1-28.4$ \\
\hline DIPSI by capillary glucose $(n=99)$ & 2 hours $\geq 140$ & 25 & 25.3 & $17.7-34.6$ \\
\hline
\end{tabular}

Table 3: Comparison of IADPSG with DIPSI venous samples for diagnosis of GDM.

\begin{tabular}{|c|c|c|c|c|}
\hline \multirow{2}{*}{ DIPSI capillary GDM $(\mathbf{n}=99)$} & \multicolumn{2}{|l|}{ IADPSG-GDM } & \multirow{2}{*}{ Chi square } & \multirow{2}{*}{ p-valuc } \\
\hline & Positive $(\mathrm{n}=17)$ & Negative $(n=82)$ & & \\
\hline Positive $(\mathrm{n}=25)$ & $12(70.6 \%)$ & $13(15.9 \%)$ & \multirow{2}{*}{22.349} & \multirow{2}{*}{$<0.001$} \\
\hline Negative $(n=74)$ & $5(29.4 \%)$ & $69(84.1 \%)$ & & \\
\hline
\end{tabular}

Table 4: Comparison of IADPSG with DIPSI capillary sample for diagnosis of GDM.

\begin{tabular}{|lllll|}
\hline DIPSI capillary GDM $(\mathbf{n}=\mathbf{9 9})$ & IADPSG-GDM & & Chi square & p-value \\
\hline Positive $(\mathrm{n}=25)$ & Positive $(\mathbf{n}=\mathbf{1 7})$ & Negative $(\mathbf{n}=\mathbf{8 2})$ & & \\
\hline Negative $(\mathrm{n}=74)$ & $12(70.6 \%)$ & $13(15.9 \%)$ & 22.349 & $<0.001$ \\
\hline
\end{tabular}

Among the study population $41(40.20 \%)$ women were primigravida. $9(8.82 \%)$ participants were obese, 18 $(17.65 \%)$ had family history of diabetes mellitus, 4 $(3.92 \%)$ had bad obstetric history, 1 (0.98\%) had macrosomia in previous pregnancy.

Of the 102 women screened, 18 (17.6\%) were diagnosed to have GDM by the IADPSG criteria. Screening with DIPSI criteria using venous samples and DIPSI criteria using capillary samples identified $20(19.6 \%)$ and 25 $(25.3 \%)$ women to have GDM respectively (Table 2).

Among the 18 women diagnosed as GDM by IADPSG criteria, only $13(72.2 \%)$ were diagnosed as GDM by DIPSI criteria using venous samples and remaining 5 $(27.8 \%)$ GDM women were labelled as non-diabetic. Among the 84 women diagnosed as non-diabetic by IADPSG criteria, 7 (8.3\%) women were falsely labelled as diabetic by DIPSI criteria using venous samples. The difference in the proportion of GDM women as diagnosed by two criteria was statistically significant. (p value < 0.001) (Table 3 ).

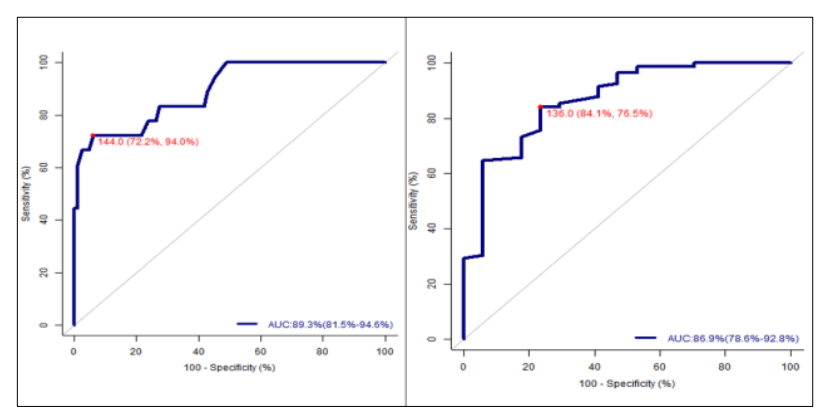

Figure 1: Predictive value of DIPSI venous and capillary sample in predicting IADPSG-GDM.

Of the 99 women studied for capillary blood samples, 17 women were diagnosed as GDM by IADPSG criteria, only $12(70.6 \%)$ were diagnosed as GDM by DIPSI 
criteria using capillary blood samples and remaining 5 $(29.4 \%)$ women were labelled as non-diabetic. Among the 82 women diagnosed as non-diabetic by IADPSG criteria $13(15.9 \%)$ women were falsely labelled as diabetic by DIPSI criteria using capillary blood samples. The difference in the proportion of GDM women as diagnosed by two criteria was statistically significant (pvalue < 0.001) (Table 4).
The DIPSI venous sample had good predictive validity in predicting IADPSG GDM, as indicated by the value of 0.893 (95\% CI 0.0 to 0.809 , p-value $<0.001$ ) for area under the Receiver Operating Characteristic (ROC) Curve. The DIPSI capillary sample also had good predictive validity in predicting IADPSG GDM, as indicated by area under the curve of 0.869 (95\% CI 0.000 to 0.774 , p-value < 0.001 ) (Figure 1 ).

Table 5: Predictive validity of DIPSI venous and capillary sample in predicting IADPSG diagnosed GDM.

\begin{tabular}{|c|c|c|c|c|c|c|}
\hline \multirow{3}{*}{ Parameter } & \multicolumn{3}{|c|}{ DIPSI venous sample $(n=102)$} & \multicolumn{3}{|c|}{ DIPSI capillary sample $(\mathrm{n}=99)$} \\
\hline & \multirow{2}{*}{ Value } & \multicolumn{2}{|c|}{$95 \%$ CI } & \multirow{2}{*}{ Value } & \multicolumn{2}{|c|}{$95 \% \mathrm{CI}$} \\
\hline & & Lower & Upper & & Lower & Upper \\
\hline Sensitivity & $72.22 \%$ & $46.52 \%$ & $90.31 \%$ & $70.59 \%$ & $44.04 \%$ & $89.69 \%$ \\
\hline Specificity & $91.67 \%$ & $83.58 \%$ & $96.58 \%$ & $84.15 \%$ & $74.42 \%$ & $91.28 \%$ \\
\hline False positive rate & $8.33 \%$ & $3.42 \%$ & $16.42 \%$ & $15.85 \%$ & $8.72 \%$ & $25.58 \%$ \\
\hline False negative rate & $27.78 \%$ & $9.69 \%$ & $53.48 \%$ & $29.41 \%$ & $10.31 \%$ & $55.96 \%$ \\
\hline Positive predictive value & $65.00 \%$ & $40.78 \%$ & $84.61 \%$ & $48.00 \%$ & $27.80 \%$ & $68.69 \%$ \\
\hline Negative predictive value & $93.90 \%$ & $86.34 \%$ & $97.99 \%$ & $93.24 \%$ & $84.93 \%$ & $97.77 \%$ \\
\hline Diagnostic accuracy & $88.24 \%$ & $80.35 \%$ & $93.77 \%$ & $81.82 \%$ & $72.80 \%$ & $88.85 \%$ \\
\hline Positive likelihood ratio & 8.67 & 1.38 & 18.305 & 4.45 & 1.15 & 9.355 \\
\hline Negative likelihood ratio & 0.30 & 0.09 & 0.64 & 0.35 & 0.07 & 0.734 \\
\hline
\end{tabular}

Based on venous blood sample, DIPSI criteria had a sensitivity of $72.22 \%$ (95\% CI $46.52 \%$ to $90.31 \%$ ) in diagnosing GDM, when compared to IADPSG criteria. Specificity by venous blood sampling was $91.67 \%$. Using capillary blood sample, DIPSI criteria had a sensitivity of $70.59 \%$ (95\% CI $44.04 \%$ to $89.69 \%$ ) in diagnosing GDM, when compared to IADPSG criteria. Specificity by capillary blood sampling was $84.15 \%$. The regression analysis $\mathrm{p}$-value of $<0.001$ indicates high proportional bias between the two measurements. The single measures intra class correlation of 0.745 (95\% CI 0.645 to 0.82 ) indicates moderate level of agreement between the capillary and venous blood glucose values (Table 5).

\section{DISCUSSION}

Diabetes mellitus (DM) a is chronic metabolic disorder characterized by hyperglycemia resulting from defects in insulin secretion, insulin action or both. There seems to be an increasing prevalence of GDM. ${ }^{10}$ Screening for GDM is usually done at 24-28 weeks of gestation because insulin resistance increases during the second and third trimester and glucose levels rise in women who do not have the ability to produce enough insulin to adapt to this resistance. ${ }^{11}$ The two main diagnostic criteria that are used for the diagnosis of GDM are the IADPSG Criteria and in India the government recommends DIPSI criteria. $^{12}$

In the current study the prevalence of GDM based on IADPSG criteria was $17.6 \%(n=18)$. The prevalence of
GDM based on DIPSI criteria using venous blood samples was $19.6 \%(n=20)$ and based on DIPSI criteria using capillary blood samples was $25.3 \%(n=25)$. Using DIPSI criteria for the diagnosis of GDM, is going to hike up the real prevalence rates of GDM in the country.

Using IADPSG criteria as the gold standard, DIPSI criteria using venous samples wrongly labelled $8.3 \%$ women as GDM, and DIPSI criteria using capillary samples wrongly labelled $15.9 \%$ women as GDM. This highlights the problem of false-positive cases diagnosed by the DIPSI criteria adding to the prevalence rates.

More worrisome is the problem of missing out the genuine GDM cases when DIPSI criteria is used. DIPSI criteria using venous blood samples had a sensitivity of $72.22 \%$, and in effect $27.8 \%$ women were labelled as false-negative. DIPSI criteria using capillary blood samples had a sensitivity of $70.59 \%$, and $29.4 \%$ women were labelled as false-negative. When DIPSI criteria is used as a universal screening modality for antenatal women, there is a definite possibility that about one quarter of women with GDM are missed, leading to catastrophic consequences for these women and their neonates.

Similar observations were also made by Srinivasan et al, Mohan et al, Tripathi et al and Hearth et al suggesting that DIPSI method had low sensitivity as well as a low positive predictive value (PPV) leading to overdiagnosis. ${ }^{13-16}$ Hence, these studies suggest that the 
use of DIPSI criteria for diagnosing GDM must be reconsidered.

ROC curves are used to compare the diagnostic performance of two or more laboratory tests. Accuracy is measured by the area under the ROC curve. An area of 1 represents a perfect test; an area of 0.5 represents a worthless test. A rough guide for classifying the accuracy of a diagnostic test is the traditional academic point system: $0.90-1=$ excellent, $0.80-0.90=$ good, $0.70-0.80=$ fair, $0.60-0.70=$ poor and $0.50-0.60=$ fail. The area measures 'discrimination', that is, the ability of the test to correctly classify those with and without the disease. DIPSI criteria is classified as 'good'. In the case of a highly prevalent disorder like GDM, any test that is less than 'excellent' should not be acceptable, due to the associated severe adverse consequences for the mother and the neonate.

One of the assumptions on which the DIPSI guidelines were framed was that it is difficult to get pregnant women to come on an empty stomach for a fasting OGTT 17. In this study out of 106 pregnant women, 96.2\% $(n=102)$ women came back in the fasting state for OGTT. Similar observations were made based on a large-scale study on Indian women from south India. ${ }^{14}$ Hence, it is reasonable to assume that once women are properly counselled, the compliance for returning back on an empty stomach for a fasting OGTT would improve due to better motivation.

In many parts of rural India, getting venous blood samples may not be possible due to lack of trained manpower or lack of facility. Some authors have suggested using capillary blood sample with glucometer may be used for the diagnosis of GDM. ${ }^{18,19}$ However, the sensitivity and specificity of capillary blood samples are unsatisfactory. Capillary blood samples should not replace venous blood samples for diagnosis of GDM by any criteria.

The DIPSI criteria for screening and diagnosis of GDM may still have a place in 'low resource settings', for which it was originally recommended. That is, where patients are not able to return back to the clinic in a fasting state for blood test, probably due to financial constraints, or non-availability of trained phlebotomists and lack of standardized laboratories to do blood glucose estimations. Another reason cited for adopting DIPSI criteria is that it is economical and convenient as only one blood sample has to be drawn. With the improvement of health care delivery in India, these pockets of 'low resource settings' in India are limited to small remote areas of rural India and they too are constantly shrinking in size. Most of the 'progressive' India of today, has adequate resources and educated women, and it would be justifiable to recommend a fasting $75 \mathrm{~g}$ OGTT as advised by IADPSG for these pregnant mothers. Although, it needs additional blood draws and may increase the cost marginally, this would be justified considering that it picks up a greater number of women with GDM compared to the DIPSI criteria.

One of the limitations of this study is that the data is from a single, tertiary care center in rural Bengaluru, but it is unlikely that this would have affected the conclusions drawn from the study. Secondly, this study had limited number of study participants.

\section{CONCLUSION}

This study demonstrates that the DIPSI criteria would miss a substantial number of patients with high risk pregnancies due to GDM. We suggest that the IADPSG criteria is better for diagnosis of GDM in antenatal women in India. Current DIPSI guidelines for India may need to be revisited.

\section{Funding: No funding sources \\ Conflict of interest: None declared}

Ethical approval: The study was approved by the Institutional Ethics Committee

\section{REFERENCES}

1. Alfadhli EM. Gestational diabetes mellitus. Saudi Med J. 2015;36(4):399-406.

2. Kampmann U, Madsen LR, Skajaa GO, Iversen DS, Moeller N, Ovesen P. Gestational diabetes: a clinical update. World J Diabetes. 2015;6(8):1065-72.

3. Lee KW, Ching SM, Ramachandran V, Yee A, Hoo FK, Chia YC, et al. Prevalence and risk factors of gestational diabetes mellitus in Asia: a systematic review and meta-analysis. BMC Preg Childbirth. 2018;18(1):494.

4. Rani PR, Begum J. Screening and diagnosis of gestational diabetes mellitus, where do we stand. J Clin Diagn Res. 2016;10(4):QE01-QE4.

5. Chi C, Loy SL, Chan SY, Choong C, Cai S, Soh SE, et al. Impact of adopting the 2013 World Health Organization criteria for diagnosis of gestational diabetes in a multi-ethnic Asian cohort: a prospective study. BMC Pregnancy Childbirth. 2018;18(1):69.

6. Balaji V, Balaji M, Anjalakshi C, Cynthia A, Arthi T, Seshiah V. Diagnosis of gestational diabetes mellitus in Asian-Indian women. Indian J Endocrinol Metab. 2011;15(3):187-90.

7. Li KT, Naik S, Alexander M, Mathad JS. Screening and diagnosis of gestational diabetes in India: a systematic review and meta-analysis. Acta Diabetol. 2018;55(6):613-25.

8. Brown FM, Wyckoff J. Application of one-step IADPSG versus two-step diagnostic criteria for gestational diabetes in the real world: impact on health services, clinical care, and outcomes. Curr Diab Rep. 2017;17(10):85.

9. Agarwal MM, Dhatt GS, Punnose J, Koster G. Gestational diabetes: dilemma caused by multiple international diagnostic criteria. Diabet Med. 2005;22(12):1731-6. 
10. Ferrara A. Increasing prevalence of gestational diabetes mellitus: a public health perspective. Diabetes Care. 2007;30 Suppl 2:S141-6.

11. Kuo CH, Chen SC, Fang CT, Nien FJ, Wu ET, Lin SY, et al. Screening gestational diabetes mellitus: The role of maternal age. PLoS One. 2017;12(3):15.

12. Mishra S, Bhadoria AS, Kishore S, Kumar R. Gestational diabetes mellitus 2018 guidelines: An update. J Family Med Prim Care. 2018;7(6):1169-72.

13. Srinivasan S, Reddi RP. Comparative study of DIPSI and IADPSG criteria for diagnosis of GDM. Int $\mathrm{J}$ Reprod Contracept Obstet Gynecol. 20148;7(3):933.

14. Mohan V, Mahalakshmi MM, Bhavadharini B, Maheswari K, Kalaiyarasi G, Anjana RM, et al. Comparison of screening for gestational diabetes mellitus by oral glucose tolerance tests done in the non-fasting (random) and fasting states. Acta Diabetol. 2014;51(6):1007-13.

15. Tripathi R, Verma D, Gupta VK, Tyagi S, Kalaivani $\mathrm{M}$, Ramji S, et al. Evaluation of $75 \mathrm{~g}$ glucose load in non-fasting state [Diabetes in Pregnancy Study group of India (DIPSI) criteria] as a diagnostic test for gestational diabetes mellitus. Indian J Med Res. 2017;145(2):209.

16. Herath $M$, Weerarathna $\mathrm{TP}$, Umesha $\mathrm{D}$. Is non fasting glucose challenge test sensitive enough to diagnose gestational diabetes mellitus? Int Arch Med. 2015;8:93.

17. Seshiah V. National Guidelines to Diagnose GDM. J Clin Diabetol. 2015;2:2.

18. Suresh Babu G, Babu Rao R, Shwetha K, Imran AS, Ratna Kumari V. Comparing the glucose values by glucometer and laboratory methods in the diagnosis of gestational diabetes mellitus: A hospital-based study. Int J Cur Med Apl Sci. 2015;7(1):21-9.

19. Jadhav DS, Wankhede UN. Comparative study of capillary blood glucose estimation by glucometer and venous plasma glucose estimation in women undergoing the one step DIPSI test (diabetes in pregnancy study group India) for screening and diagnosis of gestational diabetes mellitus. Int $\mathbf{J}$ Reprod Contracept Obstet Gynecol. 2017;6:1488-92.

Cite this article as: Saxena RK, Ansari NFT, Singh P. Effectiveness of diabetes in pregnancy study group India diagnostic criterion in detecting gestational diabetes mellitus: a rural Bangalore study. Int J Reprod Contracept Obstet Gynecol 2020;9:601-6. 